\title{
Dedekind Sums with Roots of Unity and Their Reciprocity Law
}

\author{
Chizuru SEKINE
}

Tsuda College

(Communicated by S. Fukuhara)

\section{Introduction}

For positive integers $k$ and $h$ with $\operatorname{gcd}(k, h)=1$, the classical Dedekind sum $s(k, h)$ is defined by

$$
s(k, h)=\sum_{a=1}^{h-1} \bar{B}_{1}\left(\frac{a}{h}\right) \bar{B}_{1}\left(\frac{k a}{h}\right)
$$

where $\bar{B}_{1}(x)$ is the first Bernoulli function. For $x$ real, the $n$-th Bernoulli function $\bar{B}_{n}(x)$ is defined by

$$
\bar{B}_{n}(x)=B_{n}(\{x\}) \quad \text { if } n>1 \quad \text { and } \quad \bar{B}_{1}(x)= \begin{cases}B_{1}(\{x\}) & \text { if } x \notin \mathbf{Z} \\ 0 & \text { if } x \in \mathbf{Z}\end{cases}
$$

where $\{x\}$ denotes the fractional part of $x$. The most famous property of Dedekind sum is the reciprocity law

$$
s(k, h)+s(h, k)=\frac{1}{12}\left(\frac{k}{h}+\frac{h}{k}+\frac{1}{k h}\right)-\frac{1}{4}
$$

(see [6]). Various people generalized this sum and obtained their reciprocity laws.

In [5] we focused on Apostol's generalized sum

$$
s_{n}(k, h)=\sum_{a=1}^{h-1} \frac{a}{h} \bar{B}_{n}\left(\frac{k a}{h}\right)
$$

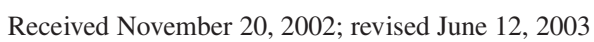


and gave another proof of the reciprocity law by using values at non-positive integers of Barnes' double zeta function $\tilde{\zeta}_{2}(s ;(k, h))$ defined by

$$
\tilde{\zeta}_{2}(s ;(k, h))=\sum_{\substack{m, n=0 \\(m, n) \neq(0,0)}}^{\infty} \frac{1}{(k m+h n)^{s}} .
$$

By the same idea, we obtained the reciprocity laws for shifted Dedekind sums, Dedekind sums with Dirichlet characters, and multiple Dedekind sums by using modified Barnes' zeta functions for each case.

In this paper, as another generalization, we obtain the reciprocity law for Dedekind sums with roots of unity (Theorem 3.3 in Section 3). For positive integers $k, h$ with $\operatorname{gcd}(k, h)=1$ and a real number $\gamma$ with $\gamma \in \frac{1}{k h} \mathbf{Z}$, Barnes' zeta function $\tilde{\zeta}_{2}(s ;(k, h), \gamma)$ with parameters $\gamma$ and $(k, h)$ is defined by

$$
\tilde{\zeta}_{2}(s ;(k, h), \gamma)=\sum_{\substack{m, n=0 \\(m, n) \neq(0,0)}}^{\infty} \frac{e^{2 \pi i \gamma(k m+h n)}}{(k m+h n)^{s}} .
$$

From the value $\tilde{\zeta}_{2}(1-n ;(k, h), \gamma)$, the reciprocity law for

$$
s_{n}(\gamma ;(k, h))=k^{n-1} \sum_{a=0}^{k-1} \sum_{b=0}^{h-1} \frac{b}{h} e^{2 \pi i \gamma(h a+k b)} \bar{B}_{n}\left(\frac{a}{k}+\frac{b}{h}\right)
$$

is obtained. This sum coincides with Apostol's when $\gamma$ is an integer.

The Dedekind sum which involves roots of unity has been considered by Berndt in [2,3], where he proved his reciprocity law by two methods, one using the transformation formulas of the generalized Eisenstein series

$$
G(z, s ; r, h)=\sum_{m, n=-\infty}^{\infty} \frac{e^{2 \pi i\left(m h_{1}+n h_{2}\right)}}{\left(\left(m+r_{1}\right) z+n+r_{2}\right)^{s}},
$$

and the other considering the contour integral of

$$
F(z)=\cot \left(\pi\left(z+\frac{j}{k h}\right)\right) \cot (\pi h z) \cot (\pi k z) .
$$

His sum and ours are related by

$$
s_{\alpha, \beta}(k, h)=s_{1}\left(\frac{j}{k h} ;(k, h)\right)-\frac{1}{2} B_{1, \frac{j}{k h}}+\frac{1}{2} B_{1, \frac{j}{k}},
$$

so we can say that our sum is the extension of his to any positive integer $n$ (see Section 4 for details). 
In Section 2 we review Barnes' multiple zeta functions, and in Section 3 we prove our reciprocity law for Dedekind sums with roots of unity. In Section 4, we relate our sums to Berndt's.

\section{Preliminary results}

In this section, we review some results which will be used in subsequent sections.

DEFINITION 2.1. The Bernoulli numbers $B_{n}$ and the Bernoulli polynomials $B_{n}(u)$ are defined by

$$
\frac{t}{e^{t}-1}=\sum_{n=0}^{\infty} \frac{B_{n}}{n !} t^{n} \quad \text { and } \quad \frac{t e^{x t}}{e^{t}-1}=\sum_{n=0}^{\infty} \frac{B_{n}(x)}{n !} t^{n}
$$

The difference equation

$$
B_{n}(x+1)=B_{n}(x)+n x^{n-1} \quad \text { if } n \geq 1
$$

holds. Also it is well-known that for a positive integer $N$, the Bernoulli function $\bar{B}_{n}$ satisfies

$$
\sum_{i=0}^{N-1} \bar{B}_{n}\left(x+\frac{i}{N}\right)=\frac{\bar{B}_{n}(N x)}{N^{n-1}}
$$

Especially,

$$
\sum_{i=0}^{N-1} \bar{B}_{n}\left(\frac{i}{N}\right)=\frac{B_{n}}{N^{n-1}}+\eta,
$$

where $\eta=1 / 2$ when $n=1$ and $\eta=0$ otherwise.

DEFINITION 2.2. Let $r$ be a positive integer and $\alpha, \omega_{1}, \cdots, \omega_{r}$ be complex numbers with positive real parts, and set $\tilde{\omega}=\left(\omega_{1}, \cdots, \omega_{r}\right)$. Barnes' $r$-ple zeta function $\zeta_{r}(s ; \alpha, \tilde{\omega})$ with parameters $\alpha$ and $\tilde{\omega}$ is defined by

$$
\zeta_{r}(s ; \alpha, \tilde{\omega})=\sum_{m_{1}, \cdots, m_{r}=0}^{\infty} \frac{1}{\left(\alpha+\omega_{1} m_{1}+\cdots+\omega_{r} m_{r}\right)^{s}} \quad \text { for } \operatorname{Re}(s)>r,
$$

and Barnes' $r$-ple zeta function $\tilde{\zeta}_{r}(s ; \tilde{\omega})$ with parameters $\tilde{\omega}$ by

$$
\tilde{\zeta}_{r}(s ; \tilde{\omega})=\sum_{\substack{m_{1}, \ldots, m_{r}=0 \\\left(m_{1}, \ldots, m_{r}\right) \neq(0, \ldots, 0)}}^{\infty} \frac{1}{\left(\omega_{1} m_{1}+\cdots+\omega_{r} m_{r}\right)^{s}} .
$$


Here $u^{s}=\exp (s \log u)$ and $\log u=\log |u|+i \arg u$ with $-\pi<\arg u<\pi$ for any complex number $u$ not on the non-positive real axis. Especially when $r=1$,

$$
\zeta_{1}\left(s ; \alpha,\left(\omega_{1}\right)\right)=\frac{1}{\omega_{1}^{s}} \zeta\left(s, \frac{\alpha}{\omega_{1}}\right) \quad \text { and } \quad \tilde{\zeta}_{1}\left(s ;\left(\omega_{1}\right)\right)=\sum_{m=1}^{\infty} \frac{1}{\left(\omega_{1} m\right)^{s}}=\frac{1}{\omega_{1}^{s}} \zeta(s)
$$

where $\zeta\left(s, \frac{\alpha}{\omega_{1}}\right)$ and $\zeta(s)$ are the Hurwitz zeta function and the Riemann zeta function, respectively.

$\zeta_{r}(s ; \alpha, \tilde{\omega})$ and $\tilde{\zeta}_{r}(s ; \tilde{\omega})$ are analytically continued to the whole complex plane, and for a positive integer $n$ we have

$$
\zeta_{r}(1-n ; \alpha, \tilde{\omega})=\frac{(-1)^{r}\left(B \omega_{1}+\cdots+B \omega_{r}+\alpha\right)^{n+r-1}}{n(n+1) \cdots(n+r-1) \cdot \Pi_{i=1}^{r} \omega_{i}}
$$

and

$$
\tilde{\zeta}_{r}(1-n ; \tilde{\omega})=\frac{(-1)^{r}\left(B \omega_{1}+\cdots+B \omega_{r}\right)^{n+r-1}}{n(n+1) \cdots(n+r-1) \cdot \Pi_{i=1}^{r} \omega_{i}}-\delta,
$$

where $\delta=1$ for $n=1, \delta=0$ otherwise, and

$$
\left(B \omega_{1}+\cdots+B \omega_{r}+\alpha\right)^{n+r-1}=\sum_{\substack{i_{1}+\cdots+i_{r}+i_{r+1}=n+r-1 \\ i_{1}, \cdots, l_{r} \geq 0}} \frac{(n+r-1) !}{i_{1} ! \cdots i_{r} ! i_{r+1} !} B_{i_{1}} \omega_{1}^{i_{1}} \cdots B_{i_{r}} \omega_{r}^{i_{r}} \alpha^{i_{r+1}}
$$

(see [4]).

Especially when $r=1$, these values are well-known:

$$
\zeta(1-n, \alpha)=-\frac{B_{n}(\alpha)}{n} \quad \text { and } \quad \zeta(1-n)=-\frac{B_{n}}{n}-\delta .
$$

In the next section we treat $\zeta(s)$ and $\zeta(s, \alpha)$ simultaneously, so we use the following notation :

$$
\zeta^{*}(s, \alpha)=\left\{\begin{array}{ll}
\zeta(s, \alpha) & \text { if } \operatorname{Re}(\alpha)>0 \\
\zeta(s) & \text { if } \alpha=0
\end{array} .\right.
$$

\section{Reciprocity law for Dedekind sums with roots of unity}

Throughout this section and the next section, $k$ and $h$ are relatively prime positive integers. 
Definition 3.1. Let $l$ be a positive integer and $l^{\prime}$ be an integer with $\operatorname{gcd}\left(l, l^{\prime}\right)=1$, and $\gamma=\frac{l^{\prime}}{l}$. Then $B_{n, \gamma}$ is defined by

$$
\sum_{a=1}^{l} \frac{e^{2 \pi i \gamma a} t e^{a t}}{e^{l t}-1}=\sum_{n=0}^{\infty} \frac{B_{n, \gamma}}{n !} t^{n}
$$

Then for any multiple $L$ of $l$,

$$
B_{n, \gamma}=L^{n-1} \sum_{a=1}^{L} e^{2 \pi i \gamma a} B_{n}\left(\frac{a}{L}\right) .
$$

We note that $B_{n, \gamma}$ is connected with the periodic Bernoulli functions $\mathscr{B}_{n}(x, A)$ in [3], p. 288. For a real number $x$ and a sequence of complex numbers $A=\left\{a_{n}\right\}_{-\infty<n<\infty}$ with period $k$, $\mathscr{B}_{n}(x, A)$ is given by

$$
\mathscr{B}_{n}(x, A)=k^{n-1} \sum_{j=0}^{k-1} a_{-j} \bar{B}_{n}\left(\frac{x+j}{k}\right) .
$$

When we take $a_{n}=e^{-2 \pi i \gamma n}=e^{-2 \pi i \frac{l^{\prime}}{l} n}, A$ has a period $l$ and

$$
B_{n, \gamma}=\mathscr{B}_{n}(0, A)+\eta \text {, }
$$

where $\eta$ is the same as in (2.4).

DEFinition 3.2. Let $\gamma$ be a real number with $\gamma \in \frac{1}{k h} \mathbf{Z}$. Barnes' double zeta function $\tilde{\zeta}_{2}(s ;(k, h), \gamma)$ with parameters $(k, h)$ and $\gamma$ is defined by

$$
\tilde{\zeta}_{2}(s ;(k, h), \gamma)=\sum_{\substack{m, n=0 \\(m, n) \neq(0,0)}}^{\infty} \frac{e^{2 \pi i \gamma(k m+h n)}}{(k m+h n)^{s}} \text { for } \operatorname{Re}(s)>2 .
$$

This function is analytically continued to the whole complex plane.

Also Dedekind sum $s_{n}(\gamma ;(k, h))$ with $\gamma$ is defined by

$$
s_{n}(\gamma ;(k, h))=k^{n-1} \sum_{a=0}^{k-1} \sum_{b=0}^{h-1} \frac{b}{h} e^{2 \pi i \gamma(h a+k b)} \bar{B}_{n}\left(\frac{a}{k}+\frac{b}{h}\right) .
$$

We note that if $\gamma$ is an integer, by using (2.3) for the sum over $a$,

$$
s_{n}(\gamma ;(k, h))=k^{n-1} \sum_{a=0}^{k-1} \sum_{b=0}^{h-1} \frac{b}{h} \bar{B}_{n}\left(\frac{a}{k}+\frac{b}{h}\right)=\sum_{b=0}^{h-1} \frac{b}{h} \bar{B}_{n}\left(\frac{k b}{h}\right)=s_{n}(k, h),
$$

where $s_{n}(k, h)$ is Apostol's sum.

Then the following reciprocity law holds: 
THEOREM 3.3. For a positive integer $n$ and $\gamma \in \frac{1}{k h} \mathbf{Z}$ not in $\mathbf{Z}$,

$$
\begin{gathered}
\frac{1}{n}\left\{h^{n-1} s_{n}(\gamma ;(k, h))+k^{n-1} s_{n}(\gamma ;(h, k))\right\}=\frac{B_{n+1, \gamma}}{h k(n+1)}+\frac{B_{n, \gamma}}{n} \\
+\sum_{a=0}^{k-1} \sum_{b=0}^{h-1} e^{2 \pi i \gamma(h a+k b)} \frac{(B k h+B k h+h a+k b)^{n+1}}{n(n+1)(k h)^{2}}-\delta,
\end{gathered}
$$

where $\delta=1$ for $n=1$ and $\delta=0$ otherwise, and we expand $(B k h+B k h+h a+k b)^{n+1}$ as in (2.9). Especially when $n=1$,

$$
\begin{aligned}
& s_{1}(\gamma ;(k, h))+ s_{1}(\gamma ;(h, k))=\frac{B_{2, \gamma}}{2 h k}+B_{1, \gamma} \\
&+\left\{\begin{array}{l}
\frac{k}{2 h} B_{2, \gamma k}-\frac{1}{2} B_{1, \gamma k}-\frac{1}{2} \\
\frac{h}{2 k} B_{2, \gamma h}-\frac{1}{2} B_{1, \gamma h}-\frac{1}{2} \\
B_{1, \gamma k} B_{1, \gamma h}-B_{1, \gamma k}-B_{1, \gamma h}
\end{array} \quad \text { if } \gamma \in \frac{1}{h} \mathbf{Z}\right. \\
&
\end{aligned}
$$

PROOF. By setting $m=b+h m^{\prime}$ and $n=a+k n^{\prime}$, and varying $a$ from 0 to $k-1$ and $b$ from 0 to $h-1$, respectively, we have

$$
\tilde{\zeta}_{2}(s ;(k, h), \gamma)=\sum_{\substack{m, n=0 \\(m, n) \neq(0,0)}}^{\infty} \frac{e^{2 \pi i \gamma(k m+h n)}}{(k m+h n)^{s}}=\sum_{a=0}^{k-1} \sum_{b=0}^{h-1} \sum_{m^{\prime}, n^{\prime}=0}^{\infty} \frac{e^{2 \pi i \gamma(h a+k b)}}{\left(h a+k b+h k\left(m^{\prime}+n^{\prime}\right)\right)^{s}}
$$

where $\sum^{\prime}$ means that we sum over all pairs of non-negative integers $\left(m^{\prime}, n^{\prime}\right)$ except for $\left(m^{\prime}, n^{\prime}\right)=(0,0)$ when $a=b=0$. Set $m^{\prime}+n^{\prime}=N$, and we have

$$
\begin{aligned}
\tilde{\zeta}_{2}(s ;(k, h), \gamma)= & \sum_{a=0}^{k-1} \sum_{b=0}^{h-1} \sum_{N=0}^{\infty} \frac{e^{2 \pi i \gamma(h a+k b)}(N+1)}{(k h)^{s}\left(\frac{h a+k b}{k h}+N\right)^{s}} \\
= & \sum_{a=0}^{k-1} \sum_{b=0}^{h-1} \frac{e^{2 \pi i \gamma(h a+k b)}}{(k h)^{s}} \zeta^{*}\left(s-1, \frac{h a+k b}{k h}\right) \\
& +\sum_{a=0}^{k-1} \sum_{b=0}^{h-1}\left(1-\frac{a}{k}-\frac{b}{h}\right) \frac{e^{2 \pi i \gamma(h a+k b)}}{(k h)^{s}} \zeta^{*}\left(s, \frac{h a+k b}{k h}\right),
\end{aligned}
$$

where $\sum^{\prime \prime}$ means that we sum over all non-negative integers $N$ except for $N=0$ when $a=b=0$, and $\zeta^{*}$ is the function defined in (2.11). 
We put $s=1-n$ into (3.5) and use (2.10). Then

$$
\begin{aligned}
\tilde{\zeta}_{2}(1-n ;(k, h), \gamma)= & -\sum_{a=0}^{k-1} \sum_{b=0}^{h-1} \frac{e^{2 \pi i \gamma(h a+k b)}}{(k h)^{1-n}(n+1)} B_{n+1}\left(\frac{h a+k b}{k h}\right) \\
& -\sum_{a=0}^{k-1} \sum_{b=0}^{h-1}\left(1-\frac{a}{k}-\frac{b}{h}\right) \frac{e^{2 \pi i \gamma(h a+k b)}}{(k h)^{1-n} n} B_{n}\left(\frac{h a+k b}{k h}\right)-\delta .
\end{aligned}
$$

For the values of $a$ and $b$ in the sums of (3.6), we have $0 \leq \frac{h a+k b}{k h}=\frac{a}{k}+\frac{b}{h}<2$ and $\frac{h a+k b}{k h} \neq 1$. Also we note that

$$
\left\{\left\{\frac{h a+k b}{k h}\right\} \mid a, b \in \mathbf{Z}, 0 \leq a<k, 0 \leq b<h\right\}=\left\{\frac{i}{k h} \mid 0 \leq i \leq h k-1\right\} .
$$

Let $S$ denote the set

$$
S=\left\{(a, b) \in \mathbf{Z}^{2} \mid 0 \leq a<k, 0 \leq b<h, \frac{a}{k}+\frac{b}{h}>1\right\} .
$$

Then we can simplify the sums in (3.6) by separating the sum $\sum_{a=0}^{k-1} \sum_{b=0}^{h-1}$ into $\sum_{(a, b) \notin S}$ and $\sum_{(a, b) \in S}$, and using (2.2), (3.2), and

$$
\sum_{a=0}^{k-1} \sum_{b=0}^{h-1} e^{2 \pi i \gamma(h a+k b)} B_{n}\left(\left\{\frac{h a+k b}{k h}\right\}\right)=\sum_{j=1}^{k h} e^{2 \pi i \gamma j} B_{n}\left(\frac{j}{k h}\right)-\delta=\frac{B_{n, \gamma}}{(k h)^{n-1}}-\delta .
$$

Namely,

$$
\begin{aligned}
\sum_{a=0}^{k-1} \sum_{b=0}^{h-1} & \left(1-\frac{a}{k}-\frac{b}{h}\right) \frac{e^{2 \pi i \gamma(h a+k b)}}{(k h)^{1-n}} B_{n}\left(\frac{h a+k b}{k h}\right) \\
= & \sum_{a=0}^{k-1} \sum_{b=0}^{h-1}\left(1-\frac{a}{k}-\frac{b}{h}\right) \frac{e^{2 \pi i \gamma(h a+k b)}}{(k h)^{1-n}} B_{n}\left(\left\{\frac{h a+k b}{k h}\right\}\right) \\
& -n \sum_{(a, b) \in S} \frac{e^{2 \pi i \gamma(h a+k b)}}{(k h)^{1-n}}\left(\frac{a}{k}+\frac{b}{h}-1\right)^{n} \\
= & B_{n, \gamma}-\delta-h^{n-1} s_{n}(\gamma,(k, h))-k^{n-1} s_{n}(\gamma,(h, k)) \\
& -n \sum_{(a, b) \in S} \frac{e^{2 \pi i \gamma(h a+k b)}\left(\frac{a}{k}+\frac{b}{h}-1\right)^{n} .}{(k h)^{1-n}} .
\end{aligned}
$$

We can also calculate the first term in the right hand side of (3.6), and finally (3.6) becomes $\tilde{\zeta}_{2}(1-n ;(k, h), \gamma)=-\frac{B_{n+1, \gamma}}{h k(n+1)}-\frac{B_{n, \gamma}}{n}+\frac{1}{n}\left\{h^{n-1} s_{n}(\gamma,(k, h))+k^{n-1} s_{n}(\gamma,(h, k))\right\}$. 
On the other hand, by (2.7) and (2.8)

$$
\tilde{\zeta}_{2}(1-n ;(k, h), \gamma)=\sum_{a=0}^{k-1} \sum_{b=0}^{h-1} e^{2 \pi i \gamma(h a+k b)} \frac{(B k h+B k h+h a+k b)^{n+1}}{n(n+1)(k h)^{2}}-\delta,
$$

and therefore (3.3) is obtained.

When $n=1$, the sums over $a$ and $b$ in the right hand side of (3.3) becomes

$$
\sum_{a=0}^{k-1} \sum_{b=0}^{h-1} e^{2 \pi i \gamma(h a+k b)}\left(\frac{5}{12}-\frac{a}{k}-\frac{b}{h}+\frac{a^{2}}{2 k^{2}}+\frac{a b}{k h}+\frac{b^{2}}{2 h^{2}}\right)-1 .
$$

Now we use the following identities : For a primitive $n$-th root of unity $\zeta$,

$$
\sum_{l=0}^{n-1} \zeta^{l}=0, \quad \sum_{l=0}^{n-1} l \zeta^{l}=\frac{n}{\zeta-1}, \quad \text { and } \quad \sum_{l=0}^{n-1} l^{2} \zeta^{l}=\frac{n^{2}(\zeta-1)-2 n \zeta}{(\zeta-1)^{2}}
$$

When $\gamma \in \frac{1}{h} \mathbf{Z}$, from (3.2)

$$
\sum_{a=0}^{k-1} \sum_{b=0}^{h-1} b e^{2 \pi i \gamma(h a+k b)}=k h\left\{\sum_{b=1}^{h}\left(\frac{b}{h}-\frac{1}{2}\right) e^{2 \pi i \gamma k b}-1\right\}=k h\left(B_{1, \gamma k}-1\right) .
$$

Similarly we have

$$
\begin{aligned}
& \sum_{a=0}^{k-1} \sum_{b=0}^{h-1} b^{2} e^{2 \pi i \gamma(h a+k b)}=k h\left(B_{2, \gamma k}+h B_{1, \gamma k}-h\right), \\
& \sum_{a=0}^{k-1} \sum_{b=0}^{h-1} a b e^{2 \pi i \gamma(h a+k b)}=\frac{h k(k-1)}{2}\left(B_{1, \gamma k}-1\right),
\end{aligned}
$$

and

$$
\sum_{a=0}^{k-1} \sum_{b=0}^{h-1} e^{2 \pi i \gamma(h a+k b)}=\sum_{a=0}^{k-1} \sum_{b=0}^{h-1} a e^{2 \pi i \gamma(h a+k b)}=\sum_{a=0}^{k-1} \sum_{b=0}^{h-1} a^{2} e^{2 \pi i \gamma(h a+k b)}=0 .
$$

Therefore when $\gamma \in \frac{1}{h} \mathbf{Z}$, (3.4) is obtained. We can prove other cases of (3.4) in the same way.

\section{Relations to Berndt's Reciprocity law for Dedekind sums with roots of unity}

In $[2,3]$ Berndt defined modified Dedekind sums $s_{\alpha, \beta}(c, d)$ and $s_{\beta, \alpha}(d, c)$ which involve roots of unity, and proved their reciprocity law.

DEFINITION 4.1 ([2], p. 187 and [3], p. 303). Let $\alpha, \beta, c$ and $d$ be integers with $c>0, d \neq 0$, and $(c, d)=1$. Define $d^{-1} \in \mathbf{Z}$ by $d^{-1} d \equiv 1(\bmod c)$. Then $s_{\beta, \alpha}(d, c)$ is 
defined by

$$
s_{\beta, \alpha}(d, c)=\sum_{\nu \bmod c d} e^{2 \pi i\left(\frac{v \alpha}{c}+\frac{v \beta}{d}\right)}\left(\left(\frac{v}{c|d|}\right)\right)\left(\left(\frac{\nu d^{-1}}{c}\right)\right),
$$

where $((x))=\bar{B}_{1}(x)$ and $s_{0,0}(d, c)=s(d, c)$. Then Berndt obtained the following:

THEOREM 4.2 ([2], p. 190 and [3], p. 304). Let $\alpha$ and $\beta$ be integers such that $(\alpha, k)=$ $(\beta, h)=1$. Then

$s_{\beta, \alpha}(h, k)+s_{\alpha, \beta}(k, h)=\frac{1}{4 k h \sin ^{2}\left(\pi\left(\frac{\alpha h+\beta k}{k h}\right)\right)}-\frac{1}{4}\left(1+\cot \left(\frac{\pi \alpha h}{k}\right) \cot \left(\frac{\pi \beta k}{h}\right)\right)$.

In this section, we derive the above theorem by using Theorem 3.3.

PROOF. From (3.7),

$$
s_{\beta, \alpha}(h, k)=\sum_{a=0}^{k-1} \sum_{b=0}^{h-1} e^{2 \pi i\left(\frac{h a \alpha}{k}+\frac{k b \beta}{h}\right)} \bar{B}_{1}\left(\frac{h a+k b}{k h}\right) B_{1}\left(\frac{a}{k}\right)+\frac{1}{2} \sum_{b=0}^{h-1} e^{2 \pi i \cdot \frac{k b \beta}{h}} \bar{B}_{1}\left(\frac{b}{h}\right) .
$$

Then by setting $j=h \alpha+k \beta$, we have

$$
\begin{aligned}
s_{\beta, \alpha}(h, k) & =\sum_{a=0}^{k-1} \sum_{b=0}^{h-1} e^{2 \pi i \frac{j}{k h}(h a+k b)} \bar{B}_{1}\left(\frac{h a+k b}{k h}\right)\left(\frac{a}{k}-\frac{1}{2}\right)+\frac{1}{2} \sum_{b=0}^{h-1} e^{2 \pi i \frac{j}{h} b} \bar{B}_{1}\left(\frac{b}{h}\right) \\
& =s_{1}\left(\frac{j}{k h} ;(h, k)\right)-\frac{1}{2} B_{1, \frac{j}{k h}}+\frac{1}{2} B_{1, \frac{j}{h}},
\end{aligned}
$$

where $s_{1}\left(\frac{j}{k h} ;(h, k)\right)$ is our Dedekind sum. Thus

$$
\begin{aligned}
s_{\beta, \alpha}(h, k)+s_{\alpha, \beta}(k, h)= & s_{1}\left(\frac{j}{k h} ;(h, k)\right)+s_{1}\left(\frac{j}{k h} ;(k, h)\right) \\
& -B_{1, \frac{j}{k h}}+\frac{1}{2} B_{1, \frac{j}{h}}+\frac{1}{2} B_{1, \frac{j}{k}} \\
= & \frac{B_{2, \frac{j}{k h}}}{2 k h}+B_{1, \frac{j}{h}} B_{1, \frac{j}{k}}-\frac{1}{2} B_{1, \frac{j}{h}}-\frac{1}{2} B_{1, \frac{j}{k}},
\end{aligned}
$$

where for the second equality, we used the third case of our reciprocity law (3.4). This is because $j$ and $k h$ are relatively prime when $(\alpha, k)=(\beta, h)=1$. Now from (3.2) and (3.7), we obtain

$$
B_{1, \frac{j}{h}}=\frac{1}{e^{2 \pi i \cdot \frac{j}{h}}-1}+1=\frac{1}{2}-\frac{i}{2} \cot \frac{j}{h} \pi
$$


and

$$
B_{2, \frac{j}{k h}}=-\frac{2 e^{2 \pi i \cdot \frac{j}{k h}}}{\left(e^{2 \pi i \cdot \frac{j}{k h}}-1\right)^{2}}=\frac{1}{2 \sin ^{2} \frac{j}{k h} \pi} .
$$

From (4.4)-(4.6), we complete the proof.

ACKNOWLEDGements. The author thanks Prof. K. Ota for the suggestion of this paper and a lot of advice.

\section{References}

[ 1 ] T. Apostol, Generalized Dedekind sums and transformation formulae of certain Lambert series, Duke Math. J. 17 (1950), 147-157

[2] B. C. Berndt, Generalized Eisenstein series and modified Dedekind sums, J. Reine Angew. Math. 272 (1975), 182-193.

[ 3 ] B. C. BERndT, Reciprocity Theorems for Dedekind Sums and Generalizations, Adv. Math. 23 (1977), 285316.

[ 4 ] K. Katayama and M. Ohtsuki, On the Multiple Gamma-Functions, Tokyo J. Math. 21 (1998), 159-182.

[5] Y. NAGASAKA, K. OTA, and C. SeKine, Generalizarions of Dedekind sums and their reciprocity laws, Acta Arith., 106 (2003), 355-378.

[ 6] H. Rademacher, Topics in Analytic Number Theory, Grundlehren Math. Wiss. 169 (1973), Springer.

Present Address:

Department of Mathematics \& Computer Science, Tsuda College,

KODAIRA, TOKYO, 187-8577 JAPAN.

e-mail: m99sekin@tsuda.ac.jp 